\title{
Beneficios da terapia comunitária integrativa revelados por usuários de substâncias psicoativas
}

\author{
Benefits of Integrative Community Therapy revealed by psychoactive drug users
} Beneficios de la terapia comunitaria integrativa revelados por usuarios de sustancias psicoactivas

Alisséia Guimarães Lemes i https://orcid.org/0000-0001-6155-6473

Elias Marcelino da Rocha io https:///orcid.org/0000-0002-0086--2286

Vagner Ferreira do Nascimento io hitps://orcid.org/0000-0002-3355-163x

Rosa Jacinto Volpato io hitps:///orcid.org/0000-0001-5709-7008

Maria Aparecida Sousa Oliveira Almeida io https://orcid.org/0000-0002-1448-2923 '

Suzicléia Elizabete de Jesus Franco ib nttps://orcid.org/0000-0002-2288-9117'

Tatiane Xavier Bauer io hitps://orcid.org/0000-0002-4682-5257'

Margarita Antonia Villar Luis id hitps://orcid.org/0000-0002-9907-5146 ${ }^{2}$

\section{Como citar:}

Lemes AG, Rocha EM, Nascimento VF, Volpato RJ, Almeida MA, Franco SE, et al. Benefícios da terapia comunitária integrativa revelados por usuários de substâncias psicoativas. Acta Paul Enferm. 2020;33:e-APE20190122

DOI http://dx.doi.org/10.37689/ acta-ape/2020A00122

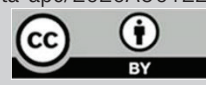

Descritores

ares; Usuários

de drogas

Keywords

User embracement; Complementary therapies; Drug ussers

Descriptores Acogimiento; Terapias complementarias; Consumidores de drogas

Submetido 14 de Maio de 2019

Aceito

18 de Julho de 2019

Autor correspondente Alisséia Guimarães Lemes https://orcid.org/0000-0001-6155-6473 E-mail: alisseia@hotmail.com

\section{Resumo}

Objetivo: Identificar os principais benefícios da Terapia Comunitária Integrativa revelados por usuários de substâncias psicoativas.

Métodos: Estudo documental, retrospectivo e qualitativo, a partir dos registros das fichas de apreciação e fechamento das rodas de Terapia Comunitária Integrativa realizadas em três instituições de recuperação de dependência de drogas, localizadas no Vale do Araguaia, Brasil. Foram apreciadas 18 fichas de registro de rodas de Terapia Comunitária Integrativa (seis registros de rodas realizadas em cada instituição) que continham o quantitativo e a descrição da apreciação dos terapeutas quanto aos principais temas, estratégias e benefícios. Para esta pesquisa, analisaram-se os benefícios percebidos pelos participantes nas rodas de Terapia Comunitária Integrativa, por meio da análise de conteúdo, na modalidade temática.

Resultados: Os principais benefícios foram agrupados de acordo com a teoria das necessidades de Maslow, permitindo identificá-los em núcleos, com exposições concretas e subjetivas, sendo divididos entre as necessidades sociais (acolhimento, comunhão, espaço de bem-estar e expectativa de futuro), estigma (aprendizado, entendimento e respeito) e realização (pertencimento e esperança).

Conclusão: Os registros apontaram que a Terapia Comunitária Integrativa possibilitou a participação ativa dos usuários, permitindo a partilha dos sofrimentos e das alegrias entre eles, apontando esta terapia como viável e acessível para a recuperação da dependência de drogas.

\section{Abstract}

Objective: To identify the main benefits of Integrative Community Therapy revealed by users of psychoactive drugs.

Method: Documentary, retrospective and qualitative study, based on the records of the Assessment and Closure Forms of the Integrative Community Therapy groups, which were conducted in three drug addiction rehab centers located in the region of the Vale do Araguaia, Brazil. The sample consisted of eighteen records of Integrative Community Therapy groups (six records at each institution), which contained the quantity of participants and the therapists' assessment of the main themes, strategies and benefits. For this research, the benefits perceived by the participants of the Integrative Community Therapy groups were analyzed through thematic content analysis.

Results: The main benefits were grouped according to Maslow's hierarchy of needs and divided into categories with concrete and subjective experiences. The benefits were divided into social needs (embracement,

'Universidade Federal de Mato Grosso, Barra do Garças, MT, Brasil

2Escola de Enfermagem, Universidade de São Paulo, Ribeirão Preto, SP, Brasil.

${ }^{3}$ Universidade do Estado de Mato Grosso, Tangará da Serra, MT, Brasil.

Conflito de interesse: artigo extraído da tese de doutorado "A Terapia Comunitária Integrativa como estratégia de intervenção psicossocial para usuários de drogas psicoativas", a ser apresentada na modalidade conjunto de artigos ao Programa de Pós-graduação em Enfermagem Psiquiátrica da Escola de Enfermagem de Ribeirão Preto, Universidade de São Paulo, Ribeirão Preto, SP, Brasil. 
communion, space of well-being and future expectations), esteem needs (learning, understanding and respect) and self-actualization needs (belonging and hope).

Conclusion: The records indicated that the Integrative Community Therapy enabled an active participation of users, allowing them to share suffering and joy with each other. The results demonstrated that this therapy is viable and affordable for drug dependency treatment.

\section{Resumen}

Objetivo: Identificar los principales beneficios de la terapia comunitaria integrativa revelados por usuarios de sustancias psicoactivas.

Métodos: Estudio documental, retrospectivo y cualitativo, a partir de los registros de las fichas de valoración y cierre de las rondas de Terapia Comunitaria Integrativa realizadas en tres instituciones de recuperación de adicciones a las drogas, ubicadas en Vale do Araguaia, Brasil. Se evaluaron 18 fichas de registro de rondas de Terapia Comunitaria Integrativa (6 registros de rondas realizadas en cada institución), con contenido cuantitativo y descripción de la valoración de los terapeutas respecto a los principales temas, estrategias y beneficios. Para este estudio, se analizaron los beneficios percibidos por los participantes en las rondas de Terapia Comunitaria Integrativa, por medio del análisis de contenido, en la modalidad temática.

Resultados: Los principales beneficios fueron agrupados de acuerdo con la teoría de las necesidades de Maslow, lo que permitió identificarlos en núcleos, con exposiciones concretas y subjetivas, y dividirlos entre necesidades sociales (acogida, comunión, espacio de bienestar y expectativa de futuro), estigma (aprendizaje, comprensión y respeto) y realización (pertenencia y esperanza).

Conclusión: Los registros indicaron que la Terapia Comunitaria Integrativa posibilitó la participación activa de los usuarios, lo que les permitió compartir los sufrimientos y las alegrías entre ellos, indicando esta terapia como viable y accesible para la recuperación de la adicción a las drogas.

\section{Introdução}

O uso de drogas psicoativas causa, na vida dos usuários, prejuízos irreversíveis no que diz respeito a aspectos pessoais, familiares e sociais, o que pode agravar ainda mais a dependência química. ${ }^{(1)}$

Essa problemática merece atenção especial dos profissionais (saúde, social, humanas, etc.) que lidam com esse contexto, destacando a necessidade da oferta de abordagens terapêuticas que acolham os usuários em suas necessidades biopsicossociais e ainda auxiliem a desenvolverem seu autocuidado.

Uma das abordagens terapêuticas que tem sido considerada como promitente é a Terapia Comunitária Integrativa (TCI). Trata-se de uma modalidade terapêutica criada em 1987, no nordeste brasileiro e, devido à sua trajetória e relevância, foi inserida no SUS na Política Nacional das Práticas Integrativas e Complementares (PNPIC), no ano de 2008. Estudos têm apontado sua relevância e seu uso no campo da saúde mental, no que diz respeito a problemas relacionados ao uso de drogas psicoativas. ${ }^{(2,3)}$

Esta modalidade terapêutica é realizada em rodas abertas e comunitárias, contando com a participação da comunidade (específica ou não) e dos terapeutas comunitários. Qualquer categoria profissional ou pessoa da comunidade pode se tornar terapeuta comunitário, mas, para isso, é necessário integrar 240 horas de capacitação (Momento de imersão teórico e prático), promovida em um dos
16 polos/centros de formação em TCI, no Brasil ou em outros países (Argentina, Chile e Equador). ${ }^{(4)}$

A TCI tem sido apontada como relevante por permitir a integração entre seus participantes e fortalecer os vínculos entre os pares, mediando o confronto das dificuldades cotidianas e o crescimento diante do processo de tratamento. ${ }^{(4)}$

Vários estudos recentes reforçam o impacto positivo dessa prática terapêutica nos diversos perfis de comunidades. ${ }^{(5-8)}$ Diante disso, a iniciativa em conduzir a TCI com pessoas em situação de dependência química em tratamento visa oferecer espaço de escuta qualificada que permita o manejo de conflitos, sofrimentos e emoçóes advindos do uso de drogas psicoativas.

No entanto, apesar da relevância e confirmação dos benefícios terapêuticos da TCI, há ainda poucos estudos que apontam sua utilização com usuários de drogas. A partir disso, este estudo teve por objetivo identificar os principais benefícios da Terapia Comunitária Integrativa revelados por usuários de substâncias psicoativas.

\section{Métodos}

\section{Tipo de estudo}

Estudo documental, retrospectivo e com abordagem qualitativa, realizado em fichas de registro de rodas de terapia comunitária realizadas em três instituiçóes de recuperação de dependência de drogas localizadas na região do Vale do Araguaia, Brasil. 


\section{Cenário onde as rodas de $\mathrm{TCI}$ foram realizadas}

A região onde as rodas de TCI foram realizadas possui como referência quatro casas terapêuticas (CT), sendo três masculinas (duas situadas na zona rural e uma na zona urbana) e uma feminina (situada na zona urbana). Intencionalmente, escolheram-se somente as instituiçóes masculinas, pois ampliaria o número de participantes com características semelhantes, e ainda pelo fato de já se estarem desenvolvendo trabalhos sistemáticos anuais com estas instituições.

As CTs possuem caráter provisório, religioso e filantrópico seguindo as orientaçóes vigentes da Portaria MS 3088/2011 e Resolução CONAD N. 01/2015, desenvolvendo cuidados exclusivos a homens maiores de 18 anos, em condiçóes clínicas estáveis, com problemas associados ao uso nocivo ou dependência de substância psicoativa. Realizam acolhimento de pessoas, de forma voluntária, por demanda espontânea, judicial ou encaminhadas por outros serviços. Possui ambiente residencial, com tratamento médio de nove meses. Sua equipe de profissionais é composta por religiosos sem formação específica e colaboradores voluntários.

As fichas referentes ao processo de intervenção realizada com a TCI pertencem ao acervo do laboratório de pesquisa "Stress, Alcoolismo e Uso de Drogas" da Escola de Enfermagem da Universidade de São Paulo (EERP/USP), Ribeirão Preto, SP, Brasil, à qual a pesquisa de doutorado "A Terapia Comunitária Integrativa como estratégia de intervenção psicossocial para usuários de drogas psicoativas" está vinculada e, por isso, foram analisadas neste cenário/local.

\section{Critérios de seleção}

Consideraram-se como critérios de inclusão todas as fichas de registros de rodas de TCI realizadas com usuários de drogas psicoativas no período de janeiro a maio de 2018 que apresentassem os registros completos da apreciação das rodas por parte dos terapeutas comunitários, sendo considerado como critério de exclusão as fichas que não foram realizadas com grupos de usuários de drogas psicoativas.

\section{Instrumentos e coleta de dados}

As fontes principais de informação foram os registros, também denominados "Ficha de Apreciação e
Fechamento das rodas", de preenchimento obrigatório pelos terapeutas comunitários, após o término de cada roda. Como trata-se de registros da equipe de terapeutas, os participantes não possuem acesso as fichas. A ficha é padrão, utilizada por todos os terapeutas comunitários do país. Ela foi criada, na década de 90, pelo idealizador da TCI, Dr. Adalberto Barreto. ${ }^{(4)}$

Nessas fichas, constam o quantitativo e a descrição da apreciaçáo dos terapeutas quanto aos principais temas apresentados e escolhidos pelos participantes, mote proposto (pergunta-chave), estratégias sugeridas pelo grupo para tais enfrentamentos e benefícios autorreferidos. ${ }^{(3)}$ Para esta pesquisa, entre os itens presentes nas fichas, analisaram-se os benefícios percebidos pelos participantes nas rodas de TCI.

Essas fichas foram preenchidas por três enfermeiros terapeutas comunitários durante a execução de um processo de intervenção realizado por meio de 18 rodas de TCI (seis em cada CT), no período de janeiro a maio de 2018, a partir da exposição de 21 usuários de substâncias psicoativas. Os três enfermeiros que preencheram as fichas foram os mesmos que realizaram as rodas e conduziram os grupos. Ambos possuíam capacitação em TCI.

Já a coleta de dados e a análise dessas fichas ocorreram no período de junho a novembro de 2018, nas dependências da EERP/USP.

\section{Análise e tratamento dos dados}

Para organização e sistematização dos dados, utilizou-se a teoria das necessidades básicas propostas por Maslow. ${ }^{(9)} \mathrm{O}$ autor subdividiu as necessidades humanas em fisiológicas (sono, alimentação, desejos sexuais, respiração, água, excreção), de segurança (segurança do corpo, do emprego, de recursos, de moralidade, da família, de saúde, da propriedade), sociais (troca afetiva, associações, aceitação, intimidade sexual, amizades, família), de estima (valorização, autoestima, respeito, confiança, conquista) e de realização (autorrealização, moralidade, criatividade, espontaneidade, solução de problemas, ausência de preconceito, aceitaçáo dos fatos). ${ }^{(9)}$

Para a análise, utilizou-se a análise de conteúdo, na modalidade temática, percorrendo-se três etapas: pré-análise, exploração do material e tratamento 
dos resultados. ${ }^{(10)}$ Esse processo gerou três categorias temáticas: "benefícios sociais percebidos nas rodas de TCI; benefícios de estima percebidos nas rodas de TCI; e benefícios de realização percebidos nas rodas de TCI".

Foram seguidas as diretrizes para realização de estudos qualitativos conforme orientação da rede Equator, pelo protocolo internacional Consolidated Criteria For Reporting Qualitative Research (COREQ). ${ }^{(11)}$ Para isso, antecipadamente se consideraram todos os critérios desse protocolo para concepção e construção do estudo, porém, por se tratar de um estudo documental, houve uma nova verificação e confirmação do cumprimento dos 15/32 itens do checklist padrão.

\section{Aspectos éticos}

Foram respeitados todos os aspectos éticos em pesquisa com seres humanos, de acordo com a Resolução 466/2012 do Conselho Nacional de Saúde (CNS), iniciando-se a investigação somente após aprovação do Comitê de Ética em Pesquisa da EERP-USP, sob CAAE: 68444017.8.0000.5393 e parecer no 2.487.000 em 06/02/2018.

\section{Resultados}

\section{Apresentação dos resultados}

Seguindo-se a teoria de Maslow, os benefícios expressivos nas primeiras necessidades básicas (fisiológicas e de segurança) não foram percebidos ou foram mencionados de forma pouco expressiva pelos participantes, entretanto, nas três seguintes (sociais, estima e realização), foi possível incluir todos os benéficos expostos nas rodas de TCI, como apresentado no quadro 1.

Uma síntese dos benefícios percebidos apresentada no quadro 2 permitiu identificá-los em núcleos com exposições mais concretamente vivenciadas e outras de natureza mais subjetiva. No caso das necessidades sociais concretas, identifica-se que as menções se concentraram em dois núcleos, no acolhimento e na comunhão, enquanto as sociais, de ordem subjetiva, se concentraram em espaço de bem-estar e expectativa de futuro.
Quadro 1. Número de citações dos benefícios percebidos pelos participantes nas rodas de Terapia Comunitária Integrativa, distribuídas de acordo com as necessidades de Maslow

\begin{tabular}{|c|c|c|c|c|}
\hline Fisiológicas & Segurança & Sociais & Estima & Realização \\
\hline \multirow{14}{*}{--} & Conforto (1) & Acolhimento (7) & $\begin{array}{l}\text { Aprendizado } \\
\text { (11) }\end{array}$ & Pertença (7) \\
\hline & \multirow{13}{*}{ Incentivo (1) } & $\begin{array}{c}\text { Comunhão/ } \\
\text { união (5) }\end{array}$ & Respeito (9) & Esperança (6) \\
\hline & & $\begin{array}{l}\text { Espaço para sentir- } \\
\text { se bem (5) }\end{array}$ & $\begin{array}{c}\text { Entendimento } \\
\text { (8) }\end{array}$ & Alegria (4) \\
\hline & & Amor (3) & $\begin{array}{c}\text { Conhecimento } \\
\text { (8) }\end{array}$ & Felicidade (4) \\
\hline & & $\operatorname{Paz}(3)$ & Experiência (5) & Gratidão (2) \\
\hline & & Recomeço (2) & Sabedoria (3) & Futuro (1) \\
\hline & & Oportunidade (2) & Apoio (2) & Desabafo (1) \\
\hline & & Companheirismo (2) & Satisfação (2) & Paciência (1) \\
\hline & & Amizade (2) & Vitória (2) & $\begin{array}{c}\text { Força de } \\
\text { vontade (1) }\end{array}$ \\
\hline & & Construção (2) & Liberdade (1) & \multirow{5}{*}{$\begin{array}{l}\text { Fortalecimentc } \\
\text { (1) }\end{array}$} \\
\hline & & $\begin{array}{l}\text { Importância do } \\
\text { relacionamento (2) }\end{array}$ & Alívio (1) & \\
\hline & & Harmonia (1) & Valorização (1) & \\
\hline & & Compromisso (1) & Perdão (1) & \\
\hline & & Perseverança (1) & Confiança (1) & \\
\hline
\end{tabular}

Quadro 2. Distribuição em núcleos dos benefícios percebidos pelos participantes nas rodas de Terapia Comunitária Integrativa, de acordo com as necessidades de Maslow

\begin{tabular}{|c|c|c|}
\hline Sociais (35) & Estima (55) & Realização (27) \\
\hline $\begin{array}{c}\text { Acolhimento (11) } \\
\text { (Amor e relacionamento) }\end{array}$ & $\begin{array}{c}\text { Aprendizado (27) } \\
\text { (Conhecimento, experiência e } \\
\text { sabedoria) }\end{array}$ & $\begin{array}{c}\text { Pertencimento (11) } \\
\text { (Gratidão, fortalecimento, } \\
\text { desabafo e paciência) }\end{array}$ \\
\hline $\begin{array}{c}\text { Comunhão (8) } \\
\text { (União, companheirismo e } \\
\text { amizade) }\end{array}$ & $\begin{array}{c}\text { Entendimento (12) } \\
\text { (Compreensão, apoio, } \\
\text { valorização e confiança) }\end{array}$ & $\begin{array}{c}\text { Esperança (16) } \\
\text { (Alegria, felicidade, futuro e } \\
\text { força de vontade) }\end{array}$ \\
\cline { 1 - 1 } $\begin{array}{c}\text { Espaço de bem-estar (9) } \\
\text { (Paz e harmonia) }\end{array}$ & $\begin{array}{c}\text { Respeito (16) } \\
\text { (Vitória, satisfação, alívio, } \\
\text { perdão e liberdade) }\end{array}$ & \\
\cline { 1 - 2 } $\begin{array}{c}\text { Expectativa de futuro (7) } \\
\text { (Recomeço, perseverança e } \\
\text { construção) }\end{array}$ & \multicolumn{2}{|c}{} \\
\hline
\end{tabular}

Conforme se percebe no quadro 2 , em relação às mençôes de estima, também foi possível concentrar algumas mençóes em torno de núcleos que as abrangeriam, como no caso dos benefícios de aprendizado, entendimento e respeito. Com exceção do primeiro núcleo, as restantes são de natureza bastante subjetiva, embora envolvam, em grande medida, o relacionamento com as outras pessoas e instituições.

No mesmo quadro (2), nas necessidades de realização, parecem predominar aqueles benefícios que valorizam as relaçóes com os outros, mediante o sentimento explícito de necessidade de pertença que constitui um núcleo que abrange outras necessidades que se atrelariam a ela. Também o núcleo da esperança que incorpora o que ela propicia no sujeito (alegria, felicidade) e, inclusive, ter expectativa 
de futuro, embora haja o reconhecimento de que não basta ter esperança, é necessário possuir força de vontade para atingir essas necessidades.

Numericamente, como aponta o quadro 2, no conjunto das citaçóes de necessidades mencionadas conforme os núcleos relativos às três necessidades básicas de Maslow identificadas, percebe-se que as necessidades relativas à estima predominaram sobre as outras ( 55 menções). O que faz sentido, considerando-se a natureza dos participantes, um grupo que vivencia o sentimento sistemático de abandono e exclusão no seu cotidiano.

Da mesma forma, nas necessidades apontadas, revela-se o desejo de adquirir conhecimentos (inclusive sobre si mesmo), incorporar experiências e acumular sabedoria. Também são perceptíveis as necessidades de ser aceito (compreensão e apoio) e, finalmente, alcançar o respeito, que envolveria o perdão (autoperdão e dos outros - família), isso confere ao sujeito possibilidade de vivenciar uma série de outras necessidades apontadas.

\section{Discussão}

\section{Benefícios sociais percebidos nas rodas de TCI}

De acordo com a teoria de Maslow, as necessidades sociais (amor, afeiçáo e relacionamento com outras pessoas) fazem parte do terceiro nível de divisão das necessidades humanas e dizem respeito à participação, aceitação por companheiros, às novas amizades, aos afetos, ao amor e relacionamento interpessoal. (9) Neste estudo, atenderam, com maior expressividade, as necessidades sociais, com destaque para os benefícios de acolhimento, comunhão, expectativas de futuro e espaço de bem-estar.

As mençóes ao acolhimento devem-se à necessidade desse perfil de usuários em expressar/receber/perceber o sentimento de integração ao grupo, uma vez que, sentir-se acolhido favorece a compreensão sobre a condição de saúde, contribui para o empoderamento e pode facilitar o enfrentamento das adversidades pessoais. ${ }^{(7)}$ Esse benefício (acolhimento) também foi reportado em um estudo que utilizou a TCI em Mato Grosso, apontando-o como um processo crucial para celebrar a vida e as oportunidades de cuidar e ser cuidado. ${ }^{(12)}$
Outras modalidades de estudo também apontam que o ser humano deve ser acolhido em todos os aspectos de sua vida, que compreendem suas necessidades humanas, com ênfase no atendimento às necessidades humanas de pessoas com uso problemático de drogas psicoativas, por apresentarem-se com grande vulnerabilidade e exigirem cuidados terapêuticos complementares. No desenvolvimento do acolhimento, as experiências danosas ligadas à drogadição tendem a ser suavizadas pela redução de medos e ansiedades, aspectos comuns da dependência química. ${ }^{(3,13)}$

Sentir-se acolhido favorece a partilha, a comunhão no grupo e, assim, no presente estudo, pesquisadoras identificaram as rodas de TCI como espaço de comunhão, com formação de vínculos, amizades e apoio coletivo, ${ }^{(14)}$ benefícios que fortalecem o cuidado terapêutico do dependente de drogas. Em situação de comunhão, as pessoas se unem em prol de um resultado em comum, tornando-se mais próximas, unidas para alcançar objetivos semelhantes, com o abandono das drogas. ${ }^{(15)}$

Além disso, o espaço oportunizado pela terapia comunitária torna-se um meio propício para a promoção de encontros da comunidade, dando-se valor às histórias de vida e às vivências. Nas rodas de TCI, é possível que os participantes tomem consciência de si e do mundo ao descobrirem que cada pessoa possui trajetórias diversas, de dor, perdas e fardos. No entanto, em meio às adversidades, a partilha produz saberes e aponta novos caminhos, ${ }^{(4)}$ o que pode ampliar a expectativa de futuro desses participantes. A importância de um espaço onde os usuários possam sentir-se aceitos também pode ser verificada em outros estudos que utilizaram a TCI como método de intervenção. ${ }^{(3,6,8)}$

As rodas de TCI, além de despertarem a confiança e o protagonismo da pessoa, promovem um ambiente terapêutico de aceitação e escuta dos problemas, o que permite aos participantes compartilharem um conjunto de estratégias de superação, dando abertura para a compreensão de outras dimensôes da vida em comunidade. ${ }^{(2,7)}$

Assim sendo, é necessário que os Terapeutas Comunitários desenvolvam habilidades para perceber as necessidades dos participantes e as acolherem, 
mostrando aceitação delas, de modo a proporcionar ajuda para que os usuários possam tomar consciência delas e, assim, contribuir com o seu tratamento.

\section{Benefícios de estima percebidos nas rodas de TCI}

Maslow aponta, em seu quarto nível, as necessidades de estima que implicam o respeito a si próprio e aos outros, envolvendo a forma com que o indivíduo se vê, avalia e compreende a autoapreciação e a autoconfiança. ${ }^{\left({ }^{9}\right)}$ Neste estudo, as necessidades de estima destacadas com maiores mençóes foram os benefícios de aprendizado, respeito e entendimento.

Quanto ao aprendizado, o usuário abusivo de drogas psicoativas pode momentaneamente possuir dificuldade de compreensão, mas a TCI contribui para a sua ampliação a partir da tomada de ciência dos saberes gerados no grupo e para a incorporaçáo da condição de "terapeuta" de si mesmo. ${ }^{(3)}$

Além disso, o usuário precisa, nos espaços de acolhimento/tratamento/reabilitação, sentir-se respeitado, isso contribui para desenvolver a confiança no tratamento e no grupo de cuidadores. Neste estudo, o respeito foi citado como benefício positivo percebido pelos usuários, assim como foi citado em estudo realizado com rodas de TCI em Cuiabá, MT, Brasil, onde seus participantes sentiam-se respeitados, a partir da menção a aspectos de um espaço que garante um diálogo respeitoso e com dignidade, baseado na troca de suas experiências. ${ }^{(12)}$

Os conhecimentos que os usuários compartilham na participação nas rodas de TCI possibilitam que eles adquiram entendimento e consciência do meio em que se inserem, seja favorável ou não, e promovem o processo de reflexão e escolha por hábitos mais saudáveis voltados a melhorar a qualidade de vida. ${ }^{(3)}$

$\mathrm{O}$ entendimento também pareceu ser notado em um estudo que usou a TCI com um paciente etilista em que as rodas foram apontadas como um momento de compreensão, reflexão e desenvolvimento pessoal. ${ }^{(2)}$

Dessa forma, o papel do terapeuta comunitário centra-se em convidar os participantes a refletirem sobre sua história, a necessidade de cuidar de si, de amar-se, para poder cuidar do outro. ${ }^{(14,15)}$ Nas rodas de TCI, deve haver estímulo à expressão de poten- cialidades dos sujeitos e à valorização da capacidade de gerar soluçóes para suas dificuldades.

\section{Benefícios de realização percebidos nas rodas de TCI}

Segundo Maslow, no quinto e último nível, as necessidades de realização estão relacionadas à satisfação dos objetivos pessoais e ao alcance pleno do potencial individual. ${ }^{(9)}$ Quanto a essas necessidades, destacaram-se, neste estudo, os benefícios que envolvem os sentimentos de pertencimento e esperança.

O sentimento de pertencimento é o que define as pessoas como participantes de uma determinada comunidade de onde vêm suas características culturais e sociais. ${ }^{(16)}$ Esse sentimento pôde ser verificado entre os participantes deste estudo, pois acredita-se que, na terapia comunitária, à medida que as pessoas vão participando, a partir da partilha de suas histórias, elas vão transformando os seus sentimentos e podem ressignificar suas vidas e criar novos laços sociais. ${ }^{(4)}$ Esse conjunto de aquisiçóes, muitas vezes, configura-se como uma experiência nova e pode ter como resultado o sentimento de pertença ao grupo que, no caso das CTs em questáo, é acentuado pelo caráter religioso das atividades.

Sentir-se pertencente a um grupo pode favorecer o estreitamento de vínculos entre pessoas. Vínculos são entendidos como saudáveis quando ligam os outros de forma positiva e reforçam os sentimentos de pertença ao grupo. $\mathrm{Na}$ área da saúde, é importante que os vínculos entre a equipe de profissionais de saúde e a comunidade sejam estabelecidos, ${ }^{(6)}$ favorecendo assim o cuidado prestado e recebido. Essa oportunidade de criar vínculos pode significar ainda a possibilidade de contar com uma rede de apoio, uma comunidade que tem interesse pelo sofrimento do outro, ${ }^{(17)}$ dessa forma, esse sentimento se traduz pela ajuda que recebe do grupo.

A integração da TCI e o meio onde o usuário se sinta aceito conduz para o sentimento de esperança. Segundo Miller, com base em outros autores, a esperança é uma força de vida multidimensional e dinâmica que se caracteriza por uma expectativa confiante, embora incerta, de alcançar um futuro bem-sucedido, realisticamente possível e significante do ponto de vista pessoal. ${ }^{(18)}$ Já para Rocha a esperança foi descrita como uma esfera psíquica, estando ainda relacionada a pulsão de vida. ${ }^{(19)}$ 
No presente estudo, a esperança foi citada como um benefício positivo presente entre os participantes, pois acredita-se que participar dessas sessóes trouxe aos usuários a expectativa que, aliada a outros benefícios percebidos, pôde contribuir para a sua continuidade no tratamento, pelo menos, durante o tempo em que as sessóes foram realizadas (seis semanas).

Parece que os usuários encontram, nas rodas de TCI, enquanto setting, e nos terapeutas, uma forma de sustentação. E criar esse espaço torna-se relevante para avivar os usuários com "discursos esperançosos" . ${ }^{(19)}$

A importância da esperança também foi verificada em estudo realizado em Juiz de Fora, MG, Brasil, revelando que a Terapia Complementar Integrativa possibilitou aos participantes vivenciar a esperança de se ter tempos melhores, pensamentos mais claros, concentração e melhora na aparência física. ${ }^{(20)}$

Além da TCI, outras práticas integrativas vêm sendo desenvolvidas com os usuários de substâncias psicoativas e trazendo benefícios, como, por exemplo, a auriculoterapia na redução desse consumo, ${ }^{(21)}$ a arteterapia na promoção da autoconsciência diante dos motivos que levaram ao consumo, logo sinalizando novas perspectivas de cuidado e adesáo a estilos de vida mais saudáveis, ${ }^{(22)}$ e a musicoterapia na redução dos níveis de estresse, ${ }^{(23)}$ na melhora da qualidade de vida e interação com o grupo ${ }^{(24)}$ e no bem estar físico e mental. ${ }^{(25)}$

Por fim, todos os sentimentos percebidos como benéficos aos participantes deste estudo serviram para apontar a técnica utilizada, a TCI, como sendo uma prática integradora e comunitária, mesmo quando realizada em locais fechados, como, por exemplo, em comunidades terapêuticas, lócus do presente estudo, que possuem uma dinâmica e organização centradas na religiosidade.

Mesmo com as restriçóes impostas pelos locais onde a TCI foi desenvolvida, ela foi capaz de despertar, entre os participantes, o empoderamento necessário, seja no enfrentamento da dependência química, do adoecimento mental seja nos sofrimentos ocasionados pelas dificuldades advindas dos problemas do dia a dia no processo de reabilitação dentro da comunidade terapêutica, por se tratar de um espaço com normas rígidas de convivência.
Ao mesmo tempo, destaca-se que a TCI pode e deve ser expandida para outros cenários de cuidado e tratamento da dependência química, tais como, Centro de Atenção Psicossocial (CAPS) Álcool e Drogas (AD), grupos de autoajuda (alcoólicos anônimos e narcóticos anônimos), bem como para todos os serviços de saúde e a comunidade em geral, por seu potencial terapêutico para as demandas de saúde mental. As limitações deste estudo estão atribuídas à utilização de apenas um tipo de fonte de dados, o que impede a generalização dos achados. Assim sendo, investigaçóes posteriores acerca dos benefícios da aplicabilidade dessa prática devem ser realizadas, estendendo-a para outros ambientes terapêuticos.

\section{Conclusão}

Destaca-se o caráter inovador deste estudo por não haver sido encontrado nenhum outro realizado em instituições fechadas destinadas ao tratamento para dependência química utilizando a terapia comunitária. Além disso, este estudo permitiu inferir os efeitos benéficos da participação nas rodas, demonstrando que a TCI é uma tecnologia de cuidado terapêutico importante a ser utilizada no redimensionamento dos conflitos, sofrimentos e emoçóes, ampliando a rede de suporte social necessária para o tratamento da dependência química. Dessa forma, a TCI pode ser destacada como uma prática de baixo custo que pode ser inserida no tratamento de usuários de substâncias psicoativas, no que diz respeito ao cuidado de sua saúde mental. Por outro lado, a pesquisa também revelou que os espaços em que a TCI foi utilizada podem constituir-se num ambiente democrático em que os usuários tenham a liberdade de se expressar e, ao mesmo tempo, possam contar com momentos de alívio do rigor das normas estabelecidas neste contexto, durante o tratamento. Além do mais, destaca-se que o uso da TCI se consolida como uma tecnologia de cuidado de possível aplicação para enfermeiros treinados, mesmo em condiçôes não tão favoráveis para a sua prática, devido ao ajuste da mesma às normas de instituições fechadas. 


\section{Agradecimentos}

Aos usuários e gestores das comunidades terapêuticas participantes do estudo. Aos colaboradores do grupo de pesquisa em saúde mental da Universidade Federal de Mato Grosso, Campus Universitário do Araguaia que auxiliaram na pesquisa: Ana Cristina Oliveira; Jucelia Moraes de Lima; Liliane Santos da Silva; Marcos Vitor Naves Carrijo; Tayla Queren dos Santos Basso; Tayane Próspero Cardoso e Wliane Nunes Silva. À Coordenação de Aperfeiçoamento de Pessoal de Nível Superior -CAPES / Novo Prodoutoral / UFMT - bolsa de doutorado de Alisséia Guimarães Lemes.

\section{Colaborações}

Lemes AG; Rocha EM; Nascimento VF; Volpato RJ; Almeida MASO; Franco SEJ; Bauer TX e Luis MAV contribuíram com a concepção do projeto, análise e interpretação dos dados, redação do artigo, revisão crítica relevante do conteúdo intelectual e aprovação da versão final a ser publicada.

\section{Referências}

1. Perrone PA. The therapeutic community for recuperation from addiction to alcohol and other drugs in Brasil: in line with or running counter to psychiatric reform?]. Cien Saude Colet. 2014;19(2):569-80. Portuguese.

2. Giffoni FA, Santos MA. Community therapy as a method to address the problem of alcohol abuse in primary care. Rev Lat Am Enfermagem. 2011;19(n.Spe):821-30.

3. Lemes AG, Nascimento VF, Rocha EM, Moura AA, Luis MA. Integrative Community Therapy as a strategy for coping with drug among inmates in therapeutic communities: documentary research. SMAD. Rev Eletrôn Saúde Mental Álcool Drog. 2017;13(2):101-8.

4. Barreto AP. Terapia comunitária: passo a passo. Fortaleza: LCR; 2008.

5. Lessa AM, Nascimento IS, Nascimento FS, Ribeiro LG. Successful experience: Implementation of Integrative and Complementary Practices in the Duque de Caxias (RJ) municipal network. Braz J Health Rev. 2017;2(4):2847-50.

6. Lucietto GC, Ribeiro RL, Silva RA, Nascimento VF. [Integrational Community Therapy: construction of autonomy of families of renaissable children]. Rev Aten Saúde (São Caetano do Sul). 208;16(58):57-62. Portuguse.

7. Matos ML, Carvalho MA, Pascoal FF, Silva ER, Ferreira Filha MO, Souza GP, et al. Integrative Community Therapy and its Meaning for Student Life: a Meeting of Experiences. Int Arch Med. 2017;10(83):1-12.
8. de Lima Silva V, de Medeiros CA, Guerra GC, Ferreira PH, de Araújo Júnior RF, de Araújo Barbosa SJ, et al. Quality of Life, Integrative Community Therapy, Family Support, and Satisfaction with Health Services Among Elderly Adults with and without Symptoms of Depression. Psychiatr Q. 2017;88(2):359-69.

9. Maslow AH. Motivación y personalidad. 2nd ed. Nova York: Harperand How; 1970.

10. Minayo MC. Sampling and saturation in qualitative research: consensuses and controversies. Rev Pesqui Qualitativa. 2017;5(7):112.

11. Tong A, Sainsbury $P$, Craig J. Consolidated criteria for reporting qualitative research (COREQ): a 32-item checklist for interviews and focus groups. Int J Qual Health Care. 2007;19(6):349-57.

12. Melo OS, Ribeiro LR, CostaAL, Urel DR. [Community impact of integrative therapy for renal patients people during session hemodialysis]. Rev Pesqui Cuid Fundam (Online). 2015;7(2):2200-14. Portuguese.

13. Gomes MA, Abrahão AL, Silva AP. Contributions to a research intervention for nursing care to drug users. Rev Pesqui Cuid Fundam (Online). 2015;7(4):3487-95. Portuguese.

14. Moura SG, Filha MO, Moreira MA, Simpson CA, Tura LF, Silva AO. Social representations of integrative community therapy by the elderly. Rev Gaucha Enferm. 2017;38(2):e55067.

15. Araújo MÂ, Girão JE, Souza KM, Esmeraldo GR, Farias FL, Souza ÂM. The Community Therapy - creating networks solidarity in a Family Health Center. Rev Port Enferm Saude Mental. 2018;19:71-6.

16. Ferreira Filha MO, Lazarte R, Barreto AP. Impact and trends of the use of Integrative Community Therapy in the production of mental health care. Rev Eletrôn Enferm. 2015;17(2):176-7.

17. Henrique BD, Reinaldo MA, Ayres LF, Lucca MS, Rocha RL. The use of crack and other drugs: the perception of family members in relation to the support network in a reference center. Cien. Saúde Colet. 2018;23(10):3453-62.

18. Fitzgerald Miller J. Hope: a construct central to nursing. Nurs Forum. $2007 ; 42(1): 12-9$.

19. Rocha Z. [Hope is not to wait, it is to walk: philosophical reflections on hope and its resonances in psychoanalytic theory and clinic]. Rev Latinoam Psicopatol Fundam. 2007;10(2):255-73. Portuguese.

20. Camargos GL, Corrêa AA. The use of auriculoacupuncture in the treatment of chemical dependence. Rev Cient. Fagoc Saúde. 2016;1:9-18.

21. Ontiveros-González ML, Casique-Casique L, Muñoz-Torres TJ. Auriculotherapy as nursing care to decrease the consumption of marijuana and cocaine. SMAD Rev Eletrôn Saúde Mental Álcool Drog. 2018;14(3):136-43.

22. Angelim SM, Valladares-Torres AC. The design "rain metaphor" as an instrument of therapeutic communication of problematic drug addiction. Rev Cient Arteterapia Cores Vida. 2019;26(1):48-57.

23. Taets GG, Jomar RT, Abreu AM, Capella MA. Effect of music therapy on stress in chemically dependent people: a quasi-experimental study. Rev Lat Am Enfermagem. 2019;27(0 e3115):e3115.

24. Mazalli J, Petreça DR, Campos R. The use of singing as a therapeutic in respiratory rehabilitation in institutionalized elderly. J Health NPEPS. 2019;4(1):31-46.

25. Mendes DS, Moraes FS, Lima G0, Silva PR, Cunha TA, Crossetti MG, et al. Benefits of integrative and complementary practices in nursing care. J Health NPEPS. 2019;4(1):302-18. 